\title{
Far-Infrared Hydrogen Lasers in the Peculiar Star MWC 349A
}

Vladimir Strelnitski, ${ }^{*}$ Michael R. Haas, Howard A. Smith, Edwin F. Erickson, Sean W. J. Colgan, and David J. Hollenbach 


\section{Far-Infrared Hydrogen Lasers in the Peculiar Star MWC 349A}

\author{
Vladimir Strelnitski, ${ }^{\star}$ Michael R. Haas, Howard A. Smith, \\ Edwin F. Erickson, Sean W. J. Colgan, David J. Hollenbach
}

Far-infrared hydrogen recombination lines $\mathrm{H} 15 \alpha$ (169.4 micrometers), $\mathrm{H} 12 \alpha$ (88.8 micrometers), and $\mathrm{H}_{10} \mathrm{\alpha}$ (52.5 micrometers) were detected in the peculiar luminous star MWC 349A from the Kuiper Airborne Observatory. Here it is shown that at least $\mathrm{H} 15 \mathrm{\alpha}$ is strongly amplified, with the probable amplification factor being greater than or about equal to $10^{3}$ and a brightness temperature that is greater than or about equal to $10^{7} \mathrm{kelvin}$. The other two lines also show signs of amplification, although to a lesser degree. Beyond $\mathrm{H} 1 \mathrm{O \alpha}$ the amplification apparently vanishes. The newly detected amplified lines fall into the laser wavelength domain. These lasers, as well as the previously detected hydrogen masers, may originate in the photoionized circumstellar disk of MWC 349A and constrain the disk's physics and structure.

Som after the invention of the maser and liser (microwate and light amplification by the stimulated emission of raddiation, respectively), natural misers with amplifications A up to $10^{1 .}$ were discosered in interstelliar and circumstellar gas clonds (1), first at centimeter walvelengths and later in the millimeter and sulmillimeter domains, dwwn to wavelengths $\lambda \approx 0.45 \mathrm{~mm}(450$ $\mu \mathrm{m})(2,3)$. The search for natural masers (or, rather, lasers) at still shorter wavelengths is severely hampered hy the opacity of our atmosphere. In fict, throughout most of the 300-to 20- $\mu$ m region, ground-hased whervations are impromithle.

Masers and lasers work on the silme physical principle. In the laberatery, the distinction mide between them is hissed (m) the technology used to create them, with the wavelength boundary lying renghly at several hundred micrometers (4). A natural dividing principle in astrophosics is the detection technolugy: the microwave ("wave detecting") technology used for masers and the uptical ("yuantum detecting") techmolegy used for lasers. This houndary renghly coincides with that hetween lahoratory malsers and hasers and

$V$ Strelnitski and $H$. A. Smith, Laboratory for Astrophysics. National Air and Space Museum, Smithsonian Instiwition. Washington, DO 20560, USA.

M. R. Haas, E. F. Erickson, D. J. Hollenbach, NASA Ames Research Center Motfett Field CA 94035-1000. USA. S. W. J. Colgan. NASA Ames. Research Center. Mottett Field CA 94035-1000, and Search for Extraterrestrial intelligence institute, 2035 Landings Drive, Mountain VIEW. CA 94043, USA.

Th whom correspondence should be addressed. also with the above-mentioned $300-\mu \mathrm{m}$ observational barrier.

There is no reason to doubt the existence of introphysical lasers. For eximple, calculitions have predicted population inversions (5) - a necessary condition for amplification-and, in some cases, the possihility of high gains (6) in the optical and near-infrared hydrogen transitions in HII regions (which are hydrogen clouds ionized hy the ultraviolet radiation of neirhy hot stars). Yet there is a puzzling lack of ohserved astrophysical lasers. In 1979, Smith ct al. (7) reported an abnormally bright line at $\lambda=4.7 \mu \mathrm{m}$ in the spectrum of the Becklin-Neugetaluer object in ()rion. The line was identified as the hydrogen Pt $\beta$ transition and, on the basis of an analysis of level populations, it was argued that it might be lasing. However, when this ohservation was repeated, the line was not as bright (8). Within the sollar system, there is strong evidence for population inver-

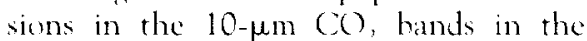
martian and venusian atmespheres (9). However, the predicted amplifications are very small $(A \leqslant 1.1)$ and are not yet sufficient to discriminate observationally hetween lasing and spontaneous fluorescence. Besides, the energies involved are $\sim 10^{15}$ times smaller than the typical enargies of the galactic masers. Thus, this local phenomenon is not the long-sought "ptical-infrared analog of the powerful, high-gain astrophysical masers.

Looking for an whject that could help solve this 30-year puzzle of why astrophysi- cal masers are ohserved and lasers are not, we chose MWC: $349 \mathrm{~A}$. This luminous star, $\approx 30,000 \mathrm{~L},(10)$, is surrounded by a massive ionized envelope that has been detectad in more than 20 hydrogen emission $\alpha$ lines from the visual $\mathrm{H} 2 \alpha$ line (1I) at $0.65 \mu \mathrm{m}$ to the radio $\mathrm{H} 92 \alpha$ line (12) at 3 coll. (Hnex denotes the hydrogen transition between the levels with principal yuantum numbers $n$ and $n+1$.) Several of the $\alpha$ lines in the millimeter domain were found to have a peculiar velecity structure: two intense, narrow spectral peaks superimposed on a broad component similar to that found for other transitions (13). The narrowness and the high intensity of the doutle-preaked component have been attributed to masing (13). The double-peaked component first appears at $\mathrm{H} 360 \mathrm{\alpha}(2.2 \mathrm{~mm})$ and is secn down to $\mathrm{H} 2 \mathrm{l} \alpha(0.45 \mathrm{~mm})$ - the shortest wavelength submillimeter $\alpha$ line observable from the ground $(2,13-16)$. Yet the optical and near-infrared $\alpha$ lines show no sign of lasing (17). Thus, MWC $349 \mathrm{~A}$ wals a promising candidate for detecting the first high-gain astrophysical lasers in the farr-infrared domain and for studying the transition from the apparently amplified long-wavelength lines $(1)$ the apparently nonamplified shortwavelength lines. The latter coukl shed light on the puzzle of the lack of ahserved lasers in generial.

We ohserved the H10 o line at $52.5 \mu \mathrm{m}$ an 15 June 1994 and the $H 12 \alpha$ and $H 15 \alpha$ lines at 88.8 and $169.4 \mu \mathrm{m}$ on 16 August 1995 with the $91-\mathrm{cm}$ telescone of the Kuiper Airhorne Ohservatory (KAO), using the facility's cryogenic gratting spectrometer $(18,19)$. Spectral resolutions were $\approx 80,80$, and $140 \mathrm{kms}$ ', respectively, and the meatsured fluxes were $36 \pm 13 \times 10^{20}, 5.5 \pm$ $2.5 \times 10^{20}$ and $8.1 \pm 1.9 \times 10^{21} \mathrm{Wcm}^{2}$, respectively. The $H 12 \alpha$ measurement is slightly allove 20 and is formally only an upper limit. The H10a line is measured to nearly 3 o and is a protahle detection (20). The $\mathrm{H} 15 \alpha$ line is more than 40 and is clearly a solid detection (Fig 1). The fact that all three lines are observed to fall within une-quarter channel of their expected wavelengths, which is well within our calibration uncertainty of \pm 0.5 channels, increases confidence in these results. No other atomic or molecular features of any significant strength are expected in any of these handpasses.

A convincing case for lasing in the newly detected lines can be made by comparing their fluxes with thesse at other wavelengths and using simple phenomenological arguments concerning the nature of the emission. The nonmasing parts of MWC 349A's envelone radiate spontaneous emission in the a lines, which is partially reahsorbed by the free electrons 
moving in the electric field of the free protons. The fluxes in the lines formed in this way are predicted to decrease with increasing $n$ according to a power law whose index varies from approximately -6 to -7 at the smallest $n$ to approximately -8 at $n \geq 40(17)$. Figure 2 compares the ohserved fluxes with the simplest spontaneons emission model - a power law with a constant "intermediate" value of the power index of -7 . The residual deviations from the model are remarkably small for two gromps of lines: the opticalnear-infrared lines fincluding the recently derected $\mathrm{H} 7 \alpha$ at $19 \mu \mathrm{m}(21) \mathrm{l}$ and the radio lines. These lines embrace, in total, almost 5 decades in wavelength and almost 12 decades in intensity. This convincingly demonstrites that all these lines (except, perhaps, H4l $\alpha$ ) are due to spontanerus cmission. In contrast, the millimeter and submillimeter lines form a prominent "hump" above the interpolated level of spontaneous emission, which confirms that they are amplified by masing.

The newly detected infrared lines also show excess radiation above the predicted spontaneous level. H1O $\alpha$ and $\mathrm{H} 12 \alpha$ were measured 14 months apart, and the nonmonotonic hehavior of their exeesses may result from variability that is similar to that ohserved for the masers in this source $(14,22)$. For buth lines, the excess is smaller than for $\mathrm{Hl} 5 \alpha$, which corrohorates the suggestion (20) that ohservahle lasing in MWC: $349 \mathrm{~A}$ vanishes somewhere near $n \approx 10$. We emphasize that the qualitative conclusion ahout a strong (more than an order of magnitude) excess in $H 15 \alpha$ does not change with the slight variations of the model power index Iseveral models were tried; see, for example, (17)]. This result is balsed on the collective properties of a large number of lines, whence its rubustness.

The detection of lasing in these lines proves that population inversions persist in the ionized recombining hydrogen down to at least $n \approx 10$ to 15 , at densities as high as $210^{4} \mathrm{~cm}^{3}$. This is in accordance with the population calculations (23) and demonstrates a hasic understanding of the numerous fundamental yuantum-mechanical processes controlling hydrogen populations at high densities.

lt is easy to explain why detectahle amplification turns on near $n \approx 40$. Past wservations have revealed two morphological components in the envelupe of MWC. 349 A: (i) an ionized outflow at $\approx 50 \mathrm{~km} \mathrm{~s}$ i (24) and (ii) a circumstellar gas-dust disk (Fig. 3). Each line has an optimum gas density at which its amplification per unit length is maximum; the lower the $n$, the higher the optimum density. Recalling that the density in the disk is proportional to $R^{3 / 2}$, where $R$ is the distance from the center, the lower the $n$ of an $\alpha$ line, the smaller should be its masing (or lasing) ring. Calculations show (17) that gains are less than unity for the lines arising from $n \geq 40$, in the peripheral part of the disk, and explain why only spontaneous emission is ohserved. For $n \leq 36$ to 40 , the gain along the ring chords first surpasses unity and then

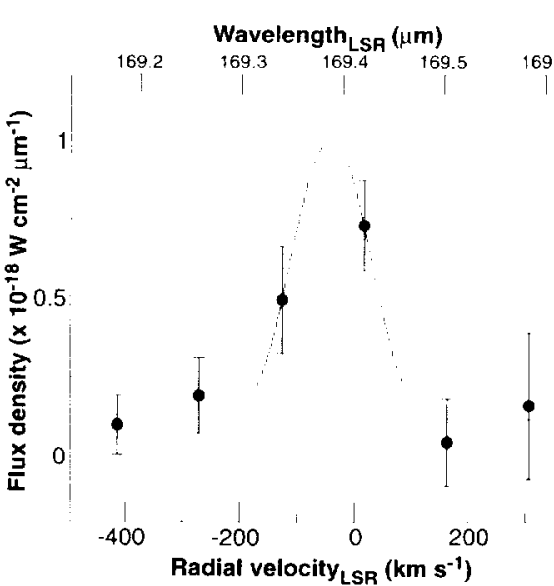

Fig. 1. The $H 15 \mathrm{c}$ spectral flux densities obtained from the KAO and the best fitted Gaussian line profile. LSR is the local standard of rest (for the stellar neighborhood of the sun).

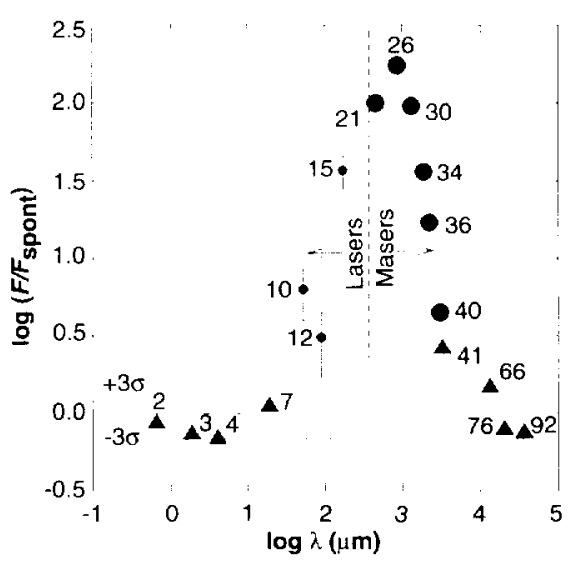

Fig. 2. A log-log plot of the ratio $F / F_{\text {congt }}$ versus $\lambda$, where $F$ is the observed integrated flux for an $x$ line of wavelength $\lambda$, and $F_{\text {i. }}=-7 \times \log n-$ 12.24. $F_{\text {: }}$ in in a least squares fit to the opticalnear-infrared and radio lines that estimates the contribution due to spontaneous emission. Triangles indicate spontaneous lines, large circles indicate masing millimeter and submillimeter lines, and small circles with error bars indicate our three KAO infrared detections. The dashed lines show \pm 30 from the fitted curve. The number near each symbol is the principal quantum number for that line's lower level. The observed fluxes in the opti$\mathrm{cal}$ and near-infrared were corrected for extinction by circumstellar and interstellar dust (30). The largest measured fluxes $(2,14,15)$ are plotted for the millimeter and submillimeter lines, which are known to be quite variable. rises toward smaller $n$. This acours even though the ring radius is decreasing-becatuse of the strong dependence of the gain
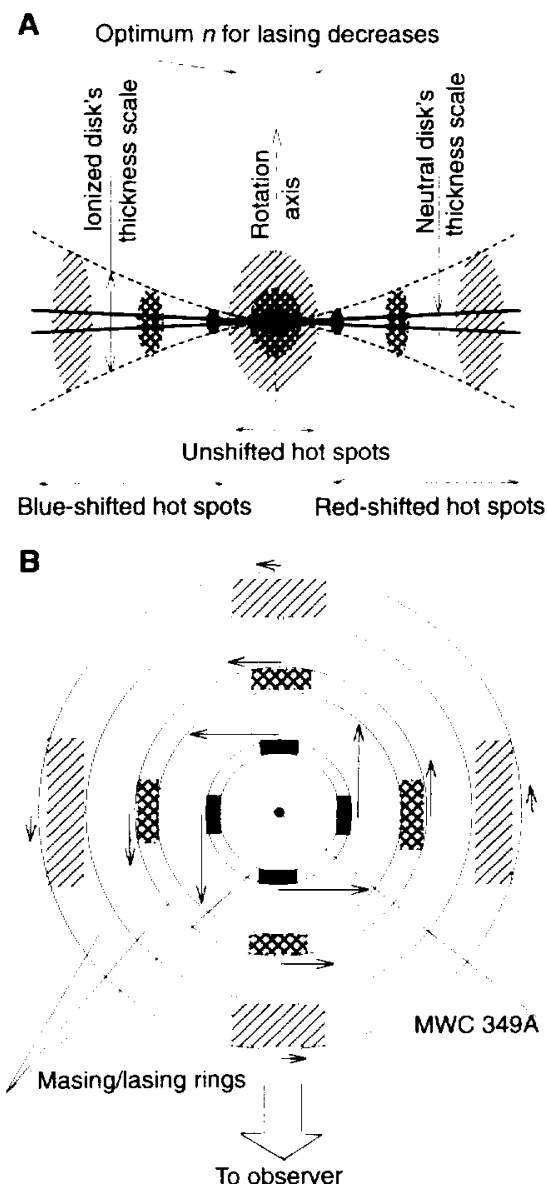

Fig. 3. The putative masing and lasing circumstellar disk in MWC 349A shown (A) edge-on ipresumably as we observe it) and (B) face-on. The double. peaked profiles of some infrared spectral lines and the masing millimeter and submillimeter hydrogen lines are ascribed to a Keplerian circumstellar disk seen edge-on $(14,31)$. According to the model (32) we adopt here. the surface layer of the gas-dust disk surrounding MWC 349A is ionized by the star's ultraviolet radiation and develops a hydrostatic $10^{t} \mathrm{~K}$ atmosphere inside a critical distance $R_{-}=200 \mathrm{AU}$. Because of decreasing gravitational binding, the atmosphere flares with distance from the star, and beyond $R$ it transforms into a stationary bipolar outflow (the outflow is not shown). The electron density in the model atmosphere is $\therefore 4 \times 10^{\circ} \mathrm{cm}$ at $R_{i}$ and is proportional to $R$ at smaller radii. The velocity vectors in $(B)$ give a rough idea of the Keplerian rotation in the disk. The sense of the rotation is still unknown: it is shown arbitrarily here. The flaring of the disk and the positions and widths of the amplifying rings are not to scale (33). The shaded areas demonstrate the paths of maximal amplification in $(B)$ and the result ing hot spots of maser and laser emission in (A) The increasing density of the shading (trom diagonal through hatched to solid toward the disk's center illustrates the increasing gas density and presumably, also the increasing brightness temperature of the masing and lasing hot spots. 
on density-and gives rise to the strongly amplified lines making the hump shown in Fig. 2.

The nore intriguing question is why does lasing apparently vanish at $n \approx 10$ ? At the moment, there are three possible explanations. (i) There is the possibility (15) that the disk only extends down to $R$ $\approx 40$ astrommical units (AU), where its ionized density is $\sim 10^{-}$to $10^{\prime \prime} \mathrm{cm}^{3}$, which is the optimum for amplification of the lines with $n \approx 20$. Becatuse the lines with lower $n$ reyuire progressively higher densities, the intensity generated in this innermost ring decreases with decreasing $n$. It is estimated (20) that in this ciase latsing hecomes undetectable near $n \approx 10$. (ii) If we assume that the disk continues inward of $\approx 40 \mathrm{AU}$ and that each lasing line forms at its optimum density, then it can ho shown (17) that for $n \leq 30$ the amplification "saturites," which slows the increase of intensity with decreasing n. Furthermore, the size of the lasing hot spot decreases with decreasing $n$ (Fig. 3), so that the flux due to spentaneous emission, which arises from the source as a whole, hecomes increasingly dominatte, and at $n$ $=10$ the lasing component becomes undetectahle on this hright sontaneous background. If this is the case, future infrared interforometers will detect small (0.1 to 0.01 are sec) bright hot spots lasing in the near-infrared hydrogen a lines in the inner disk. (iii) It is also predicted in the latter model that the laser solid angle $\Omega$ increases with $n$ as $\Omega \times n^{19}$. If the disk is cilted slighrly from our line of sight, this would remeve the low-n lasere from our view first. Future ohservations should help chouse between these (and perhaps other) possibilities. Hyputhesis (ii) - the competition berween the laser and the spontaneous emission-may provide a clue for understanding the general lack of whserved astruphysical lasers.

The brightness temperature $\left(T_{R}\right)$ and amplification of these lasers can be calculated once the ir angular sizes are known. To get a rough estimate, we assume that the disk continues inward of $=40 \mathrm{AU}$ and that HI5a forms in the disk at its optimum denity of $N \approx 1 \times 10^{\prime \prime} \mathrm{cm}$, which occurs in the mencel at a radial distance $r \approx 5 \mathrm{AU}$ from the star (17). We also alssume that H $115 x$ is a single-peaked line-any narrow double-peatied component will have an even higher brightness temperature. Taking $r$ as an upper limit to the linear scale of the laser's hot spot and assuming at line width $\Delta_{\mathrm{v}} \approx 70 \mathrm{~km} \mathrm{~s} \mathrm{~s}^{\prime}(2)$, we ohtain $T_{13} \geq\left(\lambda^{3} \mathrm{~F}\right.$ $\left.I^{2}\right) /\left(2 k \Delta v^{\prime} r^{2}\right)-10^{7} \mathrm{~K}$, where $\lambda$ is the wavelength, $k$ is Boltzmann's constant, $F$ is the shierved incegrated flux, and $D$ is the distance to the star $[\approx 1200 \mathrm{pe}(10)]$. If the input radiation temperature of the laser is eepuil to the typical physical temperature of the gas in an HIl region $\left(\sim 10^{4} \mathrm{~K}\right)$, then the amplification factor is $A \geq 10^{3}$. Similar values of $T_{B}$ and $A$ are estimated for the Hod line. Thus, these far-infrared lasers may be rather strong amplifiers, with amplification fictors and hrightness remperatures comparable to or greater than those for the millimeter masers in this source (22). Whereas the amplification in hydrogen is expected to limit gains and hrightness temperatures to less dramatic values than those for the strongest molecular masers (17), the estimated luminusities in the lasing hydrogen lines, -0.01 to 0.1 L.., are comparable to these of the strongest galactic molecular masers (25).

Detection of these new lines was due to their lasing, hecause purely spontaneous lines would be below our KAO detection thresholds. Observations with higher signal-to-noise ratios and improved spectral resoltution $\left(\leqslant 10 \mathrm{~km} \mathrm{~s}^{\prime}\right)$ are still desirable. The Infrated Satellite Oheervatory will hopefully provide higher signal-to-noise flux measurements for all the lines in the far infrared. The Stratuspheric (Ohservatury for Infrared Astronomy (26) will have sufficient spectral resulution to ohserve any double-periked velacity structure, enabling us to precisely lecate the lasers in the Keplerian disk and therehy ascertitin whether the disk has a void inward of $R=$ 40 AU, which is important for understanding planct formation in such disks (27).

\section{REFERENCES AND NOTES}

1. H. Weaver, D. R. W. Williams, N. H. Dieter, W. T. Lum. Nature 208, 29 (1965); A. C. Cheung, D. M. Rark, C. H. Townes, D. D. Thornton, W. J. Weich, ibid. 221, 626 (1969)

2. C. Thum et al. Astron. Astrophys. 288, L25 (1994).

3. K. M. Menten and K. Young. Astrophys. J. 450, L67 (1995)

4. Encyciopaedia Americana, 1984 ed. Masers; ibid. Lasers: K.-J Kim and A. Sessler, Science 250, 88 $11990)$

5. M. J. Seaton. Mon. Not. R. Astron. Soc. 119, 90 $(1959)$

6. J. H. Krolik and C. F. McKee, Astrophys. J. Stippl. Ser. 37,459 (1978).

7. H. A. Smith, H. P. Larson, U. Fink, Astrophys. J. 233 $132(1979)$.

8. N Scoville, S. Kleinmann, D. Hall, S. Ridgway, tbid. 275, 201 (1984)

9. M. J. Mumma et al. Science 212, 45 (1981); B. F Gordiets and $V$. Ya. Panchenko, Cosmic Res USA 21. 725 (1983); G. 1. Stepanova and G. M. Shved, Sov. Astron tett 11, 390 (1985): R. E. Dickinson and S. W. J. Bougher, Geophys. Res. 91, 70 1986! M. Mumma. in Astrophysical Masers. A. W. Clegg and G. E. Nedoluha, Eds. (Springer-Verlag. Berlin, 19931. p. 455.

10. M. Cohen. J. H. Bieging. J.W. Dreher, W. J. Welch, Astrophys. J. 292, 249 (1985).

11. P. W. Merrill and C. G. Burwell ibid. 78, 87 (1933).

12. L. I. Rodriguez and T. S. Bastian, ibid. 428, 324 (1994)

13. J. Martin Pintado, R. Bachiler, C. Thum, C. M. Walmsley. Astron. Astrophys, 215. L13 (1989).
14. C. Thum, J. Martin-Pintado, R. Bachiller, ibid 256 507 (1992)

15. C. Thum et al., ibid. 283, $582(1994)$

16. C. Thum, V. S. Strelnitski, J. Martin-Pintado, H. E. Matthews H. A. Smith, ibid. 300. 843 (1995).

17. V. S. Strelnitski, V. O. Ponomarev, H. A. Smith, Astrophys, $J$. in press: V. S. Strelnitski. H. A. Smith, V O. Ponomarev, bid: H. A Srrith, V. S. Strelnitski. V O. Ponomarev, in Amazing Light, R. Y. Chiao. Ed (Springer-Verlag. New York, 1996), p. 603.

18. E. F. Erickson et al. Proc. Soc. Photo-Opt instrum. Eng. 509, 129 (1984)

19. These transitions were chosen because they are relatively free of telluric absorption at $\mathrm{KAO}$ altitudes. The $\mathrm{H} 10 \mathrm{o}$ and $\mathrm{H}_{12} \mathrm{\alpha}$ lines and adjacent continua were observed with 11 Ge:St photoconductors, and the $\mathrm{H} 15 \mathrm{x}$ line and continuum were observed with six stressed Ge:Ga photoconductors. Standard chopping and nodding techniques (28) were used throughout. The raw spectra were flat-fielded with the use of Saturn observations at the same wavelengths on the same flights. The absolute flux cal bration was accomplished by multiplication of these ratioed spectra by the flux of Saturn as computed from the disk and ring contributions, with the use of a geometric model of the system (29). The total integration times for the three lines were 5200, 7440, and $4240 \mathrm{~s}$. The quoted errors of our measurements are statistical only and represent one standard deviation from the mean. The calibration uncertainty of $+25 \%$ does not substantially increase the total uncertainty.

20. V.S. Strelnitski et at., in Proceedings of the Airbome Astronomy Symposium on the Cialactic Ecosystem: From Gas to Stars to Dust, NASA. NASA Ames Re search Center, 5 to 8 July 1994. M. R. Haas, J. A. Davidson, E.F. Erickson, Eds. (Astronomical Society of the Pacitic, San Francisco, CA. 1995), p. 271

21. H. A. Smith et al. in preparation.

22. J. Martin Pintado. C. Thum, R. Bachiller, Astron. As trophys. 229. L9 (1989)

23. P. J. Storey and D. G. Hummer. Mon. Not. R. Astron Soc. 272, 41 (1995).

24. W. J. Altenhoff. P. A. Strittmatter. H. J. Wendker. Astron. Astrophys. 93, 48 (1981).

25. C. R. Gwinn. J. M. Moran. M. J. Reid Astrophys. J. 393, $149(1992)$.

26. E. F. Erickson and J. A. Davidso', in Proceedings of the Airborne Astronomy Sumposium on the Galactic Ecosystem: From Gas to Stars to Dust, M. R. Haas, J. A. Davidson, E. F. Frickson, Eds. (Astronomical Sociely of the Pacitic, San Frantisco, CA, 1995) p. 707.

27. F. H. Shu, D. Johnston, D. Hollenbach, Icarus 106. 92. (1993).

28. R. Popoular Astron. Astrophys. 117, 46 (1983)

29. B. Bezard. D. Gautier. A. Marten, ibid. 161, 387 (1986) M. R. Haas. E. F. Erickson, D. D. McKibbin, D. Goorvitch, L. J. Carott lCarus 51, 476 (1982): S. Matthews and F F Frickson NASA Technical Memorandum X.73204, p. $1(1977)$.

30. R. I. Thompson, P. A. Strittmatter. F.F. Enckson, F. L. Witteborn, D. W. Strecker, Asirophy's. J. 218, 170 (1977).

31. F. Hamann and M. Sirnon, ibid 311, 909 (1986); M A. Gordon, ibid. 387,7011992,

32. D. Hollenbach, D. Johnstone, S. Lizano, F. Shu, ibid 428, 654 (1994).

33. Laser amplification requires velocity coherence along the line of sight. In a narrow Keplerian ring seen edgeon. the coherent path is maximum along the two long est line-of-sight chords. Becalise of rotation, the chords produce two spectral cimponents that are red-shifted and blue-shifted, respectively, relative to the central star. If the ring is widk?, the gain along the central diameter can also be sufficient to produce an amplified component at zero Dospler-shift.

34. We gratelully acknowledge the KAO staff for their support of our expedition to Hawail, J. Baltz for his laboratory and flight support, anc $\mathrm{R}$. Squire for uselul comments ard for suggesting the format of Fig 3 Supported by NASA and the Simthsonian Institution. 